\title{
Modeling on Body Delay Tolerant Network Sink Locality of Wireless Body Area Networks for Different Body Postures
}

By Anthony M. Mile

Jomo Kenyatta University

Abstract- Due to the recent advancements in the field of wireless communication and Wireless Sensor Networks, the Wireless Body Area Networks (WBANs) have become an area of concern for researchers. In military operations, patient monitoring, sports field, among other wireless body area networks is used for real time monitoring and smart sensing for eHealth operations. In these WBAN, disconnections between the body sensors occur quite often and sometimes of significant duration due to the postural mobility nature of the human. These consequently affects the efficiency of the entire network hence the need for Delay Tolerant Network (DTN). The DTN minimizes delays and adapts itself to cope with long delays if they occur. One of the vital mechanisms that can be employed to enhance the efficiency of the network is to determine the optimal postural locality of the sink node.

Keywords: wireless sensor network (WSN), wireless body area network (WBAN), delay tolerant network (DTN), sink locality.

GJCST-E Classification: J.m

Strictly as per the compliance and regulations of:

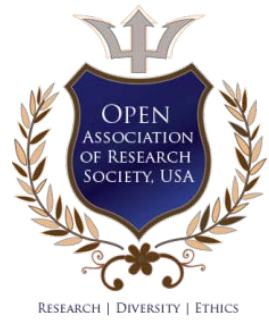

(c) 2020. Anthony M. Mile. This is a research/review paper, distributed under the terms of the Creative Commons AttributionNoncommercial 3.0 Unported License http://creativecommons.org/licenses/by-nc/3.0/), permitting all non commercial use, distribution, and reproduction in any medium, provided the original work is properly cited. 


\title{
Modeling on Body Delay Tolerant Network Sink Locality of Wireless Body Area Networks for Different Body Postures
}

\author{
Anthony M. Mile
}

Abstract- Due to the recent advancements in the field of wireless communication and Wireless Sensor Networks, the Wireless Body Area Networks (WBANs) have become an area of concern for researchers. In military operations, patient monitoring, sports field, among other wireless body area networks is used for real time monitoring and smart sensing for eHealth operations. In these WBAN, disconnections between the body sensors occur quite often and sometimes of significant duration due to the postural mobility nature of the human. These consequently affects the efficiency of the entire network hence the need for Delay Tolerant Network (DTN). The DTN minimizes delays and adapts itself to cope with long delays if they occur. One of the vital mechanisms that can be employed to enhance the efficiency of the network is to determine the optimal postural locality of the sink node. The human body experiences postural mobility leading to the WBAN topological disconnections. A WBAN model was created for illustrating and capturing on-body these disconnections. In this paper, the Omnet ++ simulation tool was used to determine the postural locality of a sink node of a human body. The results showed that the waist position of the coordinator outperforms other positions based on average delay and energy consumption. By determining the best postural locality of the sink node, among other factors, the performance and lifetime of the WBAN can be improved.

Keywords: wireless sensor network (WSN), wireless body area network (WBAN), delay tolerant network (DTN), sink locality.

\section{INTRODUCTION}

$\Lambda$ s society advances, smart healthcare services become necessary to tackle health challenges. For instance, an intelligent healthcare monitoring service can be deployed to more effectively monitor a patient from anywhere at any time (Chen et al., 2005; Darkins et al., 2008; Quwaider \& Biswas, 2008; Lv et al., 2010; Quwaider \& Biswas, 2010; Yi et al., 2016). There has been significant research which has been carried out in the last decades by both academia and industry targeted towards improved adoption and effectiveness of this technology development (Prameela \& Ponmuthuramalingam, 2016; Gowtham \& Ahila, 2017;

Author: PhD in Information Technology from Jomo Kenyatta University of Agriculture and Technology, Nairobi, Kenya and is a Scientific Computing and Computer Networks lecturer at the School of Computing and Mathematics in The Co-operative University of Kenya (CUK), Nairobi.e-mail:mileanthony@yahoo.com
Quwaider \& Biswas, 2010; Chen et al., 2011; Kumar and Singh, 2018; Mile et al., April 2018; Mile et al., June 2018; Li, 2015; Nabi et al., 2011; Bhandari \& Moh, 2016). The WBAN is a collection of wireless sensors placed on the body to collection of the physiological body signs (Arefin et al., 2017). In this Wireless Body Area Network (WBAN), the sensors node can be deployed in two possible ways: implanted and on-body sensors. In the first type of WBAN, sensor nodes are placed inside the body, whereas in the latter, sensors node are placed on the surface of body (Chen et al., 2010). Similarly, there are two ways in which data is transferred between these sensor nodes and coordinator, i.e., Point-to-Point and Multipoint-to-Point (Sipal et al., 2015). In first type, data transfer takes place between any two sensors nodes on the body, whereas in Multipoint-to-Point, data from different sensor nodes are transferred through the same sink, i.e., a coordinator forwards the data to a server located outside the body. Advancements in technology, specifically Nanotechnology and routing strategies, have made the field of Wireless Sensor Networking and specifically WBAN very attractive for researchers and developers (ul Huque et al., 2015; AL Rasyid et al., 2015).

The WBANs can be deployed in various applications in the military, sports, medicine, and healthcare, among others (Malik \& Singh, 2013; Kumar and Singh, 2018). In health monitoring, sensor nodes can be placed on a patient's body to record the patient data and send it to the coordinator node. For efficient treatment and monitoring, continuous patient monitoring is necessary. In WBAN experiencing delay challenges, this continuous monitoring may not be achievable. Another application is where underwater sensors are deployed underwater environments (Climent et al., 2014). Battlefield networks also are an application of WBAN's. In these battlefields, wireless nodes may be distributed in ad hoc topology. In the application of sensor technology in wildlife management, location and/or movement of wild animals, especially the endangered species, is monitored by implementation of sensor nodes on various parts of the body. In another adaptation of wireless sensor technology, correctional facilities adopt the technology to tracking prisoner's movement. This is achieved by implanting of the sensor nodes on the body such as ankles. The technology also 
assists keeping the security of the prisoners because it alarms when one tries to go beyond the designated safe zone.

Sensor nodes are an essential part of WBAN developers (ul Huque et al., 2015), responsible for data collection and communication with the coordinator for transfer the data. Mobility is a vital aspect of WBAN. When the body on which sensor nodes have been placed or implanted moves, the nodes on the body as well moves hence their coordination with one another changes accordingly. At one moment, nodes are well inside the communication range of one another, and at another moment, there is no connection between two nodes that were previously communicating. The human body shows postural mobility in WBAN, which causes disconnections as discussed by Mile et al. (Mile et al., April 2018; Mile et al., June 2018). In the WBAN mobility situations, instability in the WBAN sensor node connections is experienced due to mobility and distance variation, which causes the channel fading of the sensor node radio frequency.

There are always some performance parameters associated with any network to compute its efficiency. Network delay is a vital parameter in both Wireless Sensor Network (WSN) and WBAN (Jaimes \& de Sousa, 2016). In WBAN, we consider three different types of delays as propagation delay, holding or processing delay, and transmission delay. Propagation delay is the delay that the packet takes from the transmitter node to the receiver node as the source and destination nodes are apart. The propagation delay is directly proportional to the distance between the source and destination as distance and inversely proportional to the speed of data. Holding delay or processing delay is the second type of delay in WBAN and it's the processing time of data before transmission it may be due to channel busy. Transmission delay is the third type of delay in WBAN, and it is the time that the node transmits the packet to the destination. It depends on packet size and data rate. When the packet size is large, the transmission delay becomes high but when the packet size is small, transmission delay becomes small. The transmission delay is high if data rate is low and vice versa. Network average delay is some of the propagation delay, proposing delay and transmission delay. Another parameter in determining the performance of a WBAN is energy consumption, which is directly related to battery lifetime. Battery lifetime is an important parameter which defines the efficiency of the network in WBAN. Such network with a longer battery lifetime of sensor nodes with less energy consumption is more efficient compared to a network that utilizes more energy (Prameela \& Ponmuthuramalingam, 2016).

In WBAN, due to human body postural mobility, distance variation occurs from time to time between the sensor nodes due to connections drop between sensor nodes. Drops in connections can be short or long, resulting in delays in data transfer, which affects the efficiency of the entire network (Gowtham \& Ahila, 2017; Gowtham \& Ahila, 2017). Therefore, in WBAN with postural mobility, it is vital to minimize this delay and maximize network lifetime and to create a network that selects the best sink node, hence resulting to a Delay Tolerant Network (DTN). The DTNs represent the class of wireless systems that can support the functionality of a network experiencing repeated and long-term connectivity disconnects. DTN architecture improves performance and extends the range of networks having characteristics as described above. The DTN architectures are used in situations in which there is no conviction about the connection between the sensor nodes, such as underwater sensors and wildlife tracking, field networks.

In this paper, through the proposed postural selection strategy communication model, a WBAN posture selection strategy for determining the sink node locality in different human posture in a DTN is proposed. The existing models show no known adaptation of any sink node selection strategy but rather use statically selected sink nodes. By use of the Omnet++ (Varga, 2015) simulation, the performance of the DTN WBAN can be determined under the postural localities of running and sitting in order to ascertain the optimal postural locality of the sink node. The different postural locality of the sink node is evaluated in each of the mobility scenarios. The results show that the waist locality is the optimal sink position concerning the average delay and power efficiency.

The rest of this paper is organized as follows; section II discusses related work. The proposed work on the WBAN sink node selection strategy and communication model is presented in section III. Section IV presents the algorithm of the proposed postural selection strategy communication model. Section V discusses the simulation of results. Section VI discusses the results. We conclude and discuss future work in section VII.

\section{Related WORK}

A lot of work has been carried out in recent past in the field of DTN, and categorized into two, namely single-copy (Conan et al., 2008; Spyropoulos, Psounis, \& Raghavendra 2008a; Leguay et al., 2005; Jain, Fall, \& Patra, 2004) and multi-copy (Spyropoulos et al., 2008; Leguay et al., 2007; Lindgren et al., 2004). In singlecopy, a single copy of the information is transmitted to the destination node to avoid replication of data packets at destination nodes, which minimizes the replication overhead (Quwaider \& Biswas, 2010), which leads to delay minimization but it is necessary that the node is connected in their mobility. On the other hand, the multiple-copy mechanism defines how multiple copies of data packet can reach the destination node through 
different nodes, which improve the probability of packet delivery ratio (Quwaider, Tanghizadeh \& Biswas, 2011) and also increase the overall network delay which case to decrease the network lifetime.

WBAN is a short-range communication network using short transmission range for which different DTN routing mechanisms have been developed (Quwaider \& Biswas, 2012). Delivery delays of various routing techniques have been modeled. For the case of single and multi-copy DTN routing, to evaluate the performance of delay in a mobile WBAN by using a working WBAN topology which is developed and use a random posture selection without any specific information of node location for taking locations of onbody movements and applied for data packet routing which is due to the human postural mobility (Quwaider \& Biswas, 2012).

The concept of DTN also has been applied by researchers in WSNs to address the problem of intermitted network connections due to work environments and nodes behavior and to achieve power saving in WSNs by the application of Delay Tolerant Network protocol (Li, 2009).

Another research by (Quwaider, Tanghizadeh \& Biswas, 2011) presented a stochastic modeling framework for store and forward packet routing in WBAN with postural partitioning. The researchers constructed a prototype for experimentally characterizing and capturing on-body topology disconnections in the presence of ultrashort range radio links, erratic RF attenuation, and the human postural mobility. The concept was that the routing of packet or forwarding would be depended upon the posture of the human body in a WBAN. In their research, a WBANs topology was built for experimentally characterizing the disconnections of link in the presence of a human postural mobility, which then tend to develop a Delay modeling technique for evaluating single-copy on-body DTN routing protocols. This helped reduce the sensor count without losing the packet delay.

Spyropoulos et al. (Spyropoulos, Psounis, \& Raghavendra 2008) suggested flooding-based schemes to deal with networks with intermittent connectivity like the case of mobile wireless sensor networks. While flooding-based routing schemes have a high probability of delivery, they waste a lot of energy and suffer from severe contention, which can significantly degrade their performance. They are also plagued by long delays.

Vinaya Kumar (Spyropoulos et al., 2008) proposed an improved multiple copy mechanism by reducing the overhead of flooding-based schemes by introducing a new family of routing scheme that "spray" a few message copies into the network, and then route each copy independently towards the destination. They showed that, if carefully designed, spray routing not only performs significantly fewer transmissions per message.
In conclusion, while the existing single copy mechanisms do not have retransmission overheads, the data packet losses are high due to loss of end to end paths between sensor nodes and sink node leading to less efficiency due to packets loss. The mechanisms may not be ideal for WBAN, which deals with sensitive and critical data in which every single data packet may be lifesaving. On the existing multi-copy mechanisms, the flooding nature of the data packets to increase the chances of data packet delivery leads to energy waste and degraded performance. They also result into packet delays and losses due to link congestion caused by the flooding of traffic. Power saving is an important requirement of WBANs; hence these mechanisms are not ideal. In this paper, a new model was presented. The model uses an approach of dynamic sink node in which while the WBAN nodes are mobile, paths from sensor nodes to sink node are always recreated and the sink node is always reachable. This results into high reliability, high network life-time, and low power consumption of the WBAN.

\section{ili. Proposed Network Sink Locality for DTN for Different Postures}

This paper presents a postural selection strategy model for determining the sink node locality in different human posture in a DTN. A WBAN topology in Omnet ++ is implemented, to demonstrate different sink position in WBAN with different postures. In this section, we describe the WBAN architecture with different postural positions and its applications.

\section{a) Design Considerations: A WBAN Prototype and Variations in Network Topology}

In this research, twelve sensor nodes deployed on a human body to construct a WBAN architecture (node zero on the head, node one on the neck, node 2 two on left bicep, node three on right bicep, node four on the waist, node five on the left wrist, node six on right wrist, node seven on left knee, node eight on right knee, node nine on left ankle, node ten on right ankle and node eleven on the chest) as shown in Fig. 1. The CSMA/CA Mac protocol has been used in the research. A WBAN postural mobility can be described as a set of several body posture which exhibit in a human body. In this research, different experiments are performed on every posture to get the optimal position of sink node. Data communication and transmission takes place between sensor nodes and the sink node in every experiment. The responsibility of the sink node is to collect data from every sensor node and send that data to the sink node which transmits to the monitoring server via wireless link. The human body has static and mobile posture according to that, hence the WBAN architecture also changes. Mobile posture has two possibilities as running and walking, in which nodes are mobile, while in case of static posture has three possibilities as standing 
sitting and lying in which all nodes are stationary. We established and simulated both mobile and static human postures.

In this research, every posture is run for a period interval and the values of delay and energy with different sink node are recorded, which is one of the 12 nodes that communicate to the other nodes. There are certain links, that when connected during certain postures, can lead to changes in the architecture for those postures. Due to the RF attenuation, sometime disconnections occur within a posture. Fig. 1 shows the running body posture in which some parts of human body move continuously. As the leg and hand move continuously so it is the distance between the nodes as it changes time by time in running posture. Due to lot of work in running position, the human body need to send more data, hence the optimal sink selection is crucial

Walking is also a part of mobile posture, as shown in Fig. 2. In the running posture, maximum mobility was observed on legs and arms, as compared to the other nodes on the body. If we compared the running posture with walking posture, it is found that less mobility was found in the walking as compare to running. It is noted from Fig. 3, that standing posture is a static posture as classified above. Sensor nodes are deployed on the human body to collect data and communicate with the sink node. In static case, the communication between the nodes depends on the distance between the sensor nodes. When we consider that the mobility is zero, then the parameter which effects the sink location is the distance between the sink node and all other nodes. We select the node which has a maximum link with minimum distance as a sink node.

Other possible postures are sitting and lying, as shown in Figures 4 and 5 respectively. Both the sitting and lying are static postures that have no mobility and have a single architecture. In this case, all the performance metrics depend on the node distances. When we consider that the mobility is zeros, then the parameter which effects the sink location is the distance between the sink node and all other the nodes we must select the node which has the maximum link with minimum distance as a sink node.

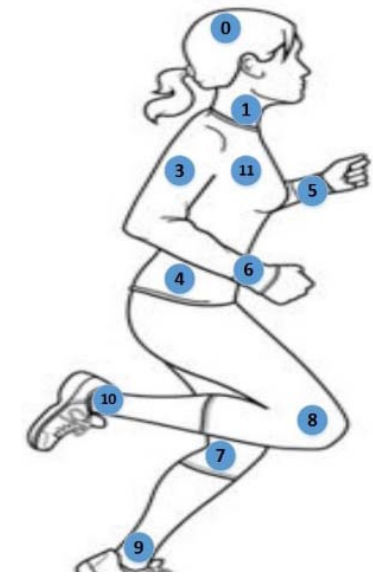

Fig. 1: Running

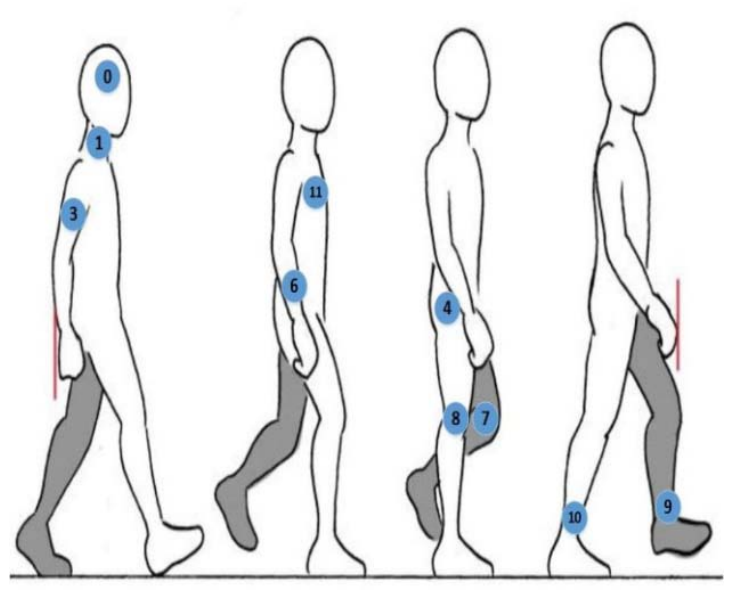

Fig. 2: Walking

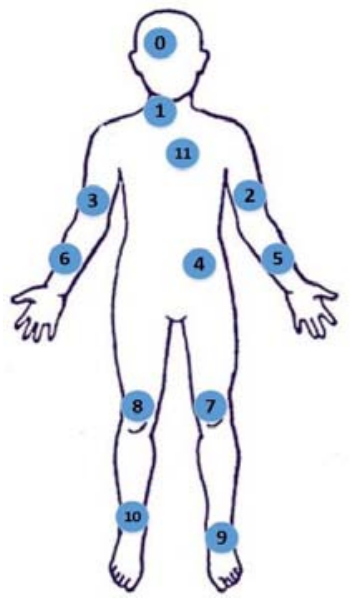

Fig. 3: Standing 


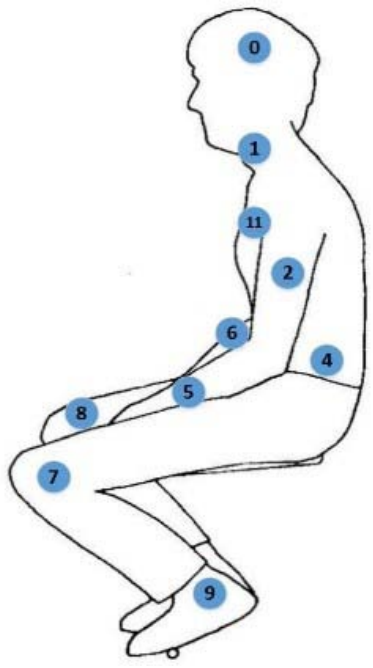

Fig. 4: Sitting

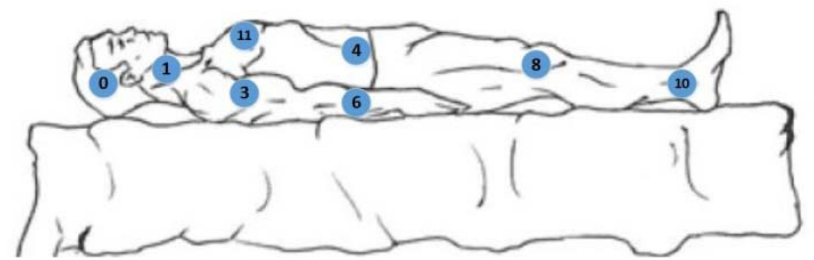

Fig. 5: Lying down

b) MoBAN Posture Selection Strategy

The human body can be in five different postures, which are broadly categorized in two ways as mobile postures and static or stationary postures. Fig. 6 shows the MoBAN posture selection scheme and its description. We consider two types of postures in our scheme, namely Mobile postures and Static postures.

In both case, whether the posture is mobile or static, the selector needs to find whether it is a mobile or a static posture in first stage. If the posture is mobile, then the target location and speed of nodes is specified by the coordinator. The node keeps moving until the destination reached.

In the static posture case, the simulation time is fixed. After time selection, the posture is selected. Once the simulation time is complete, a control is sent to the posture selector if all postures are not executed. The posture is executed until all postures are selected and then simulation ends.

c) Network Sink Locality for DTN for different Postures

In a WBAN environment, several sensors are placed on a human body for the collection of data regarding a medical condition or body movements. We created such a WBAN in Omnet++ environment composed of 12 nodes strategically placed on different body parts, as can be seen in Figures (1, 2, 3, 4, and 5). The coordinator node controls the mobility of nodes. It also performs the duty of synchronizing the movements of sensor nodes. Coordinator plays an equivalent role as the human brain in real life scenario, i.e. specifying to move, setting target position, setting the speed to move towards target position, etc. In this paper, we find the most suitable location of the sink node (one of the 12 nodes) such that minimum network resources are utilized, and a more enhanced performance network can be realized. 


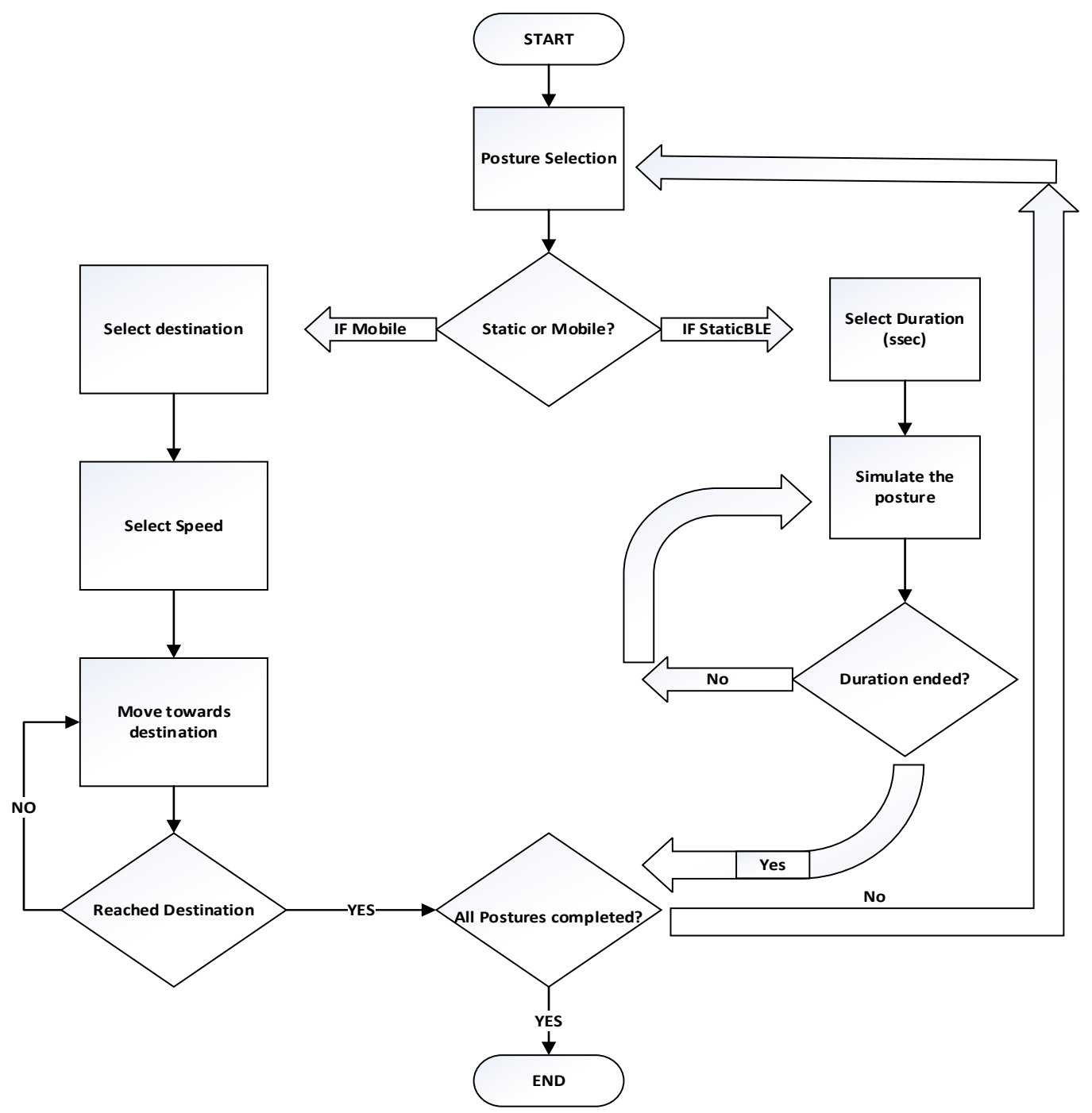

Fig. 6: MOBAN Posture Selection Strategy

The selection of suitable sink location can be critical for the performance of the network, but this is not a straight forward decision. As we select five different postures, three of them, as sitting, lying and standing are considered as stationary postures, while walking and running postures are considered as mobile postures. Greater mobility indicates high utilization of resources, i.e., battery, time, etc. In WBAN, due to postural mobility, there can be long lasting delays between different sensors. Therefore, while making a suitable selection of sink position, different factors need to be considered.

This research simulates a network for all the nodes to act as a sink and compared the performance of a network. All the sensor nodes transmit the data packet to a sink node. The sink node also sends packets to all other nodes after a specific time period to keep them alive and active.

The algorithm I describe the general flow of the research scenario. The coordinator is a component in our scenario which handles the mobility and synchronization of sensor nodes. The coordinator specifies the target position to each node and node moves to the specified location. When nodes reach their targeted positions, then the selection of the transmission node is done. Out of 12 nodes in our scenario, only one node sends the data packet at a time. The selected transmission node checks the medium availability. If it finds the medium free, it transmits its packet to the destination node. Otherwise, a back off counter is incremented. It then checks whether time allotted for transmission (transmission counter $=0.021$ seconds) to a specific node expires or not. If the transmission counter expires, then another node is selected as the transmission node. Otherwise, the selected node waits for a specific interval time then checks medium availability. Similarly, a random node is selected among receiver nodes to acknowledge the reception of packet. The selected node checks the availability of medium, and in case of free medium, it sends the acknowledgement. If the medium is unavailable, the backoff counter of the respective node is incremented. 
Then, it checks whether time allotted for acknowledgment (transmission counter $=0.021$ seconds) expires or not. If transmission counter expires, another node for acknowledging the transmission node is selected. Otherwise the selected node waits for a specific counter before it checks medium availability again. After that, it checks the simulation timer expiry. If expired, the simulation is ended. Otherwise, control is passed to the coordinator which specifies the new target position for the nodes and the whole process is repeated until the simulation timer (defined before simulation) gets expired.

\section{Algorithm of the Proposed Postural Selection Strategy Communication Model}

\section{a) The Postural Selection Strategy Communication Model}

The proposed communication model is presented in the algorithm below. It shows the flow steps from the time the coordinator position is specified until the end of simulation time.

Start simulation time specify the coordinator position Nodes move in a specific location Select transmitting node if medium available for node Transmit data wait for acknowledgement if receiver node find channel available send acknowledgement if simulation timer expires end else Go back and specify the coordinator position Repeat process else increment backoff counter if simulation timer expires Receiver node checks channel availability else wait for a specific time Receiver node checks channel availability wait for medium free else increment backoff counter if transmission counter expire select transmitting node check channel availability else wait for a specific time check channel availability End.

\section{Simulation Results}

This research has implemented basic postures of a human body as static posture (sitting) and mobile posture (running) in OMNET ++ (v.4.6) and executed a sequence of experiments to find the best location of the sink node. Data packets from sensor nodes are transferred to the destination node.

In order to avoid collisions, CSMA/CA MAC protocol is implemented so that only one node utilizes the communication channel at a time. During simulation, all nodes in a network forwards data to current sink node which transmits the data to the coordinator.

In this paper, the network delay is minimized. The network delay depends on the location of sink node. It is affected by the distance between the sensor nodes and the sink node. Energy Consumptions is another performance metric which affect the network lifetime. List of parameters used in this scenario is shown in Table 1.
Table 1: Simulation Parameters

\begin{tabular}{|c|c|}
\hline PARAMETER NAME & Value \\
\hline pMax & $110.11 \mathrm{~mW}$ \\
\hline Carrier Frequency & $2.412 \times 10^{9} \mathrm{~Hz}$ \\
\hline Alpha & 3.0 \\
\hline Saturation & $-110 \mathrm{dBm}$ \\
\hline Time Tx to Rx & $0.00012 \mathrm{~s}$ \\
\hline Time Tx to sleep & $0.000032 \mathrm{~s}$ \\
\hline Queue Length & 5 \\
\hline Header Length & $24 \mathrm{bit}$ \\
\hline Max Tx Attempts & 14 \\
\hline Bitrate & $15360 \mathrm{bps}$ \\
\hline Tx Power & $110.11 \mathrm{~mW}$ \\
\hline Sleep Current & 10 \\
\hline Tx Current & 5000 \\
\hline Rx Current & 25 \\
\hline
\end{tabular}

a) Average Delay

Delay is an important parameter describing the overall performance of the network. Therefore, we are interested in finding a sink node position which minimized the overall network delay. In WBAN, the distance between the nodes and the sink node is an important factor which determine the delay of the network. The distance between the sensor nodes and sink node change regularly due to postural mobility. Distance between a node and sink node is directly proportional to the delay. Average delay not only depend on the distance of the nodes, but also depends on the channel accessibility, i.e., less processing delay. Similarly, WBAN mobility also plays a vital role in network delay. As a mobile node changes position in the network, it also informs the rest of the nodes about its new position. Thus, as mobility increases, these positions notification packets also increases increasing the congestion and average delay of the network.

Running: The simulation was run for all the nodes as sink nodes. Some of the best choices for sink nodes (head, waist, chest, wrist, and ankle) are given in Fig. 7. The starting point of the average delay curve for every node as sink depends upon the time at which the respective node was involved in the network. It is evident from Fig. 8 that when the node the ankle was selected as the sink node due to the high mobility of ankle node the delay of network increase, the node at head compare to ankle is less mobile, so it shows a small increment in delay than ankle node while when select he node on the waist as a sink its shows optimal performance in mobile network delay due to less mobility and nearest to all other nodes. Thus, the node at waist proves to be the optimal option to be considered as sink node running posture. 
Sitting: The simulation was run for all the nodes as sink nodes. Some of the best choices considered as sink nodes (head, waist, chest, wrist, and ankle) are given in Fig. 8. The starting point for the average delay curve for every node as sink depends upon the time at which the respective node was involved in the network. Sitting posture is a static network, therefore, it does not depend on the mobility of nodes, but it depends on the distance of the nodes to the sink node as shown in Fig. 8. When we select node at the head as a sink node, due to maximum distance from other nodes it shows maximum delay when compare to the ankle and the waist node positions. When the node on waist was selected as the sink, its shows optimal performance in sitting posture because the waist position is nearest to all other nodes. Thus, node at waist proves to be the optimal option to be considered as sink node in sitting posture.

Overall Network: The simulation was run for all the nodes as sink nodes, but some of the best choices to be considered as sink nodes (head, waist, chest, wrist, and ankle) are given in Fig. 9. The starting point of the average delay curve for every node as sink depended on the time at which the respective node was involved in the network. As in overall network, we consider both the mobile posture as well as static posture. As shown in Fig. 9, the ankle position as sink node led to high delay. This can be attributed to the high mobility nature of the legs. The head position when compared to ankle position of the node, the head being less mobile than the ankle, showed slightly better delay performance than the ankle. The node on waist as a sink showed the optimal performance in overall network delay due to less mobility and nearest to all other nodes. Thus, node at waist proves to be the optimal option to be considered as sink node.

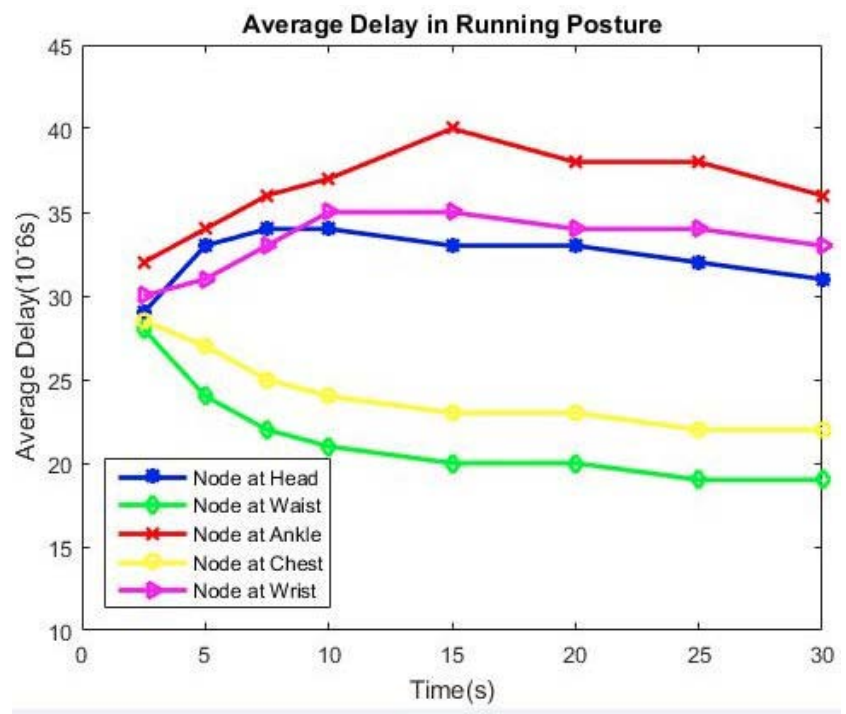

Fig. 7: Lying Average Delay in Running Posture Selection

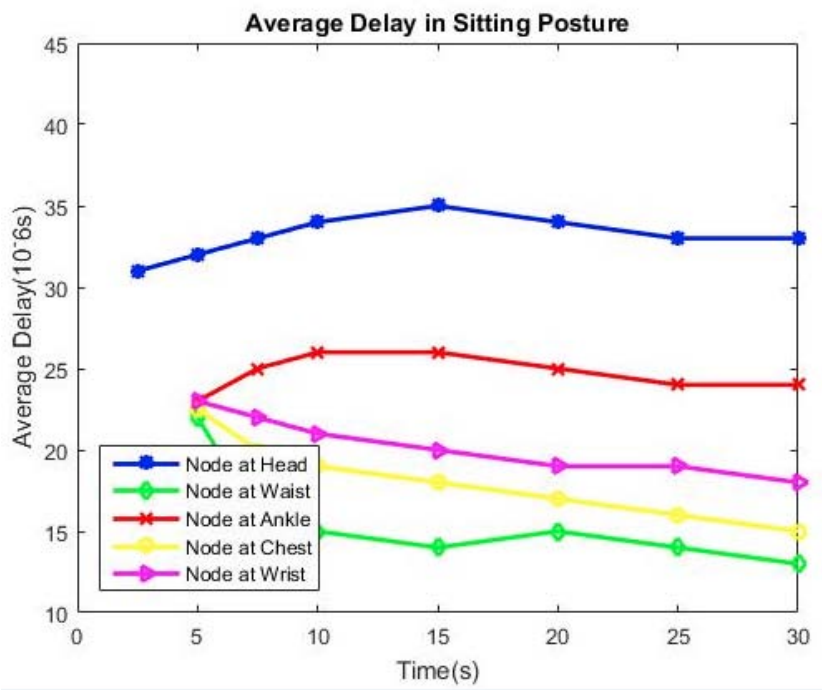

Fig. 8: Average Delay in Sitting Posture Selection

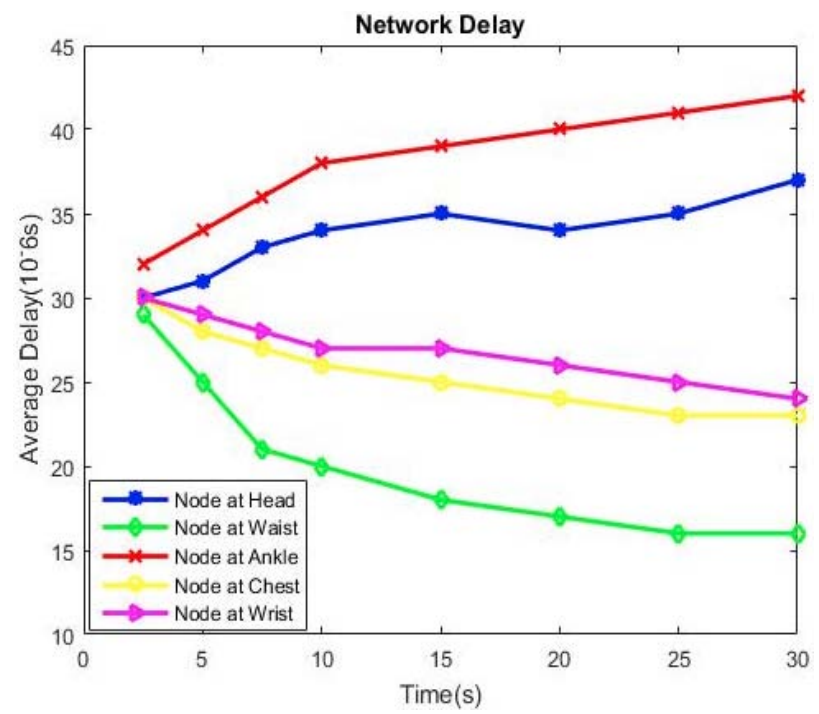

Fig. 9: Average Delay in Overall Network

\section{b) Energy Consumption in different Posture}

Battery Battery lifetime is a crucial parameter in WSNs and especially in WBAN's because some of the sensor nodes are deployed inside the body, which means they are not easily and frequently removed out. This means that the battery life is a critical feature of the sensor node. Therefore, there is need to minimize the energy consumption of sensor nodes to improve the battery lifetime and energy consumption. The battery lifetime and energy consumption are directly depended on both the node distance to the destination node and the number of retransmission. In our scheme, all the node communicates with the sink node so the distance between the sink node the other maximum node should be shorter.

In this research, the simulation was run on two different postures only, running as the mobile posture and sitting as the stationary posture. In the sitting 
posture, all the nodes were considered as stationary for comparison of different sink location as the head node, the waist node and the ankle node.

Running: The simulation was run for all the nodes as sink nodes with head, waist, chest, wrist, and ankle positions being considered. The results are shown in Fig. 10. In this posture, nodes move continuously, hence increasing the energy consumption of the sink. From Fig. 10 it is shown that energy consumption of nodes at ankle is highest than energy consumption at node deployed on the head and the waist due to the high mobility of ankle in running posture. Energy consumption also depend on the distance between the sink and the other node, the node which is nearest to the other node has least energy consumption as shown in Fig. 10 the energy consumption at the waist node is least than the other two so the node at the waist is best choice for a sink in running posture according to energy consumption.

Sitting: The simulation was run for all the nodes as sink nodes. These are the head, waist, ankle, chest and the wrist as shown in Fig. 11. Like in the Sitting posture, there is no mobility involved, the energy difference is based on the postural locality of sink node from other nodes and coordinator. The greater the distance between the sink node from other nodes and coordinator, the greater is the energy consumption of that specific node. As node at the waist was located at the center of body, its distance from the other nodes was smaller as compared to other nodes in Fig. 11. That is why energy consumption of node at waist is smaller than other nodes in Fig. 11. Therefore, we conclude that the node at waist is the ultimate choice of sink node in Sitting Posture in relation to energy consumption.

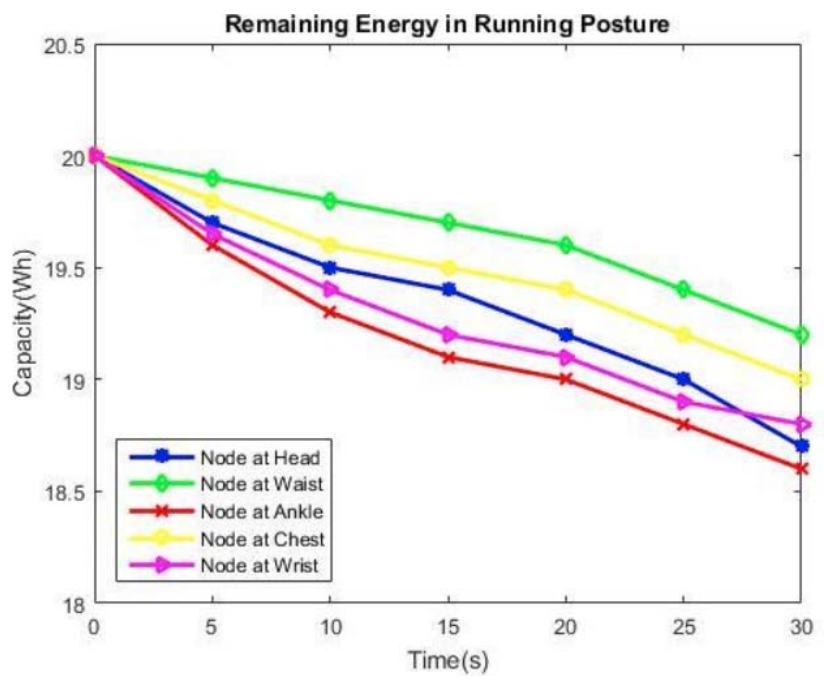

Fig. 10: Average Energy Consumption in running posture

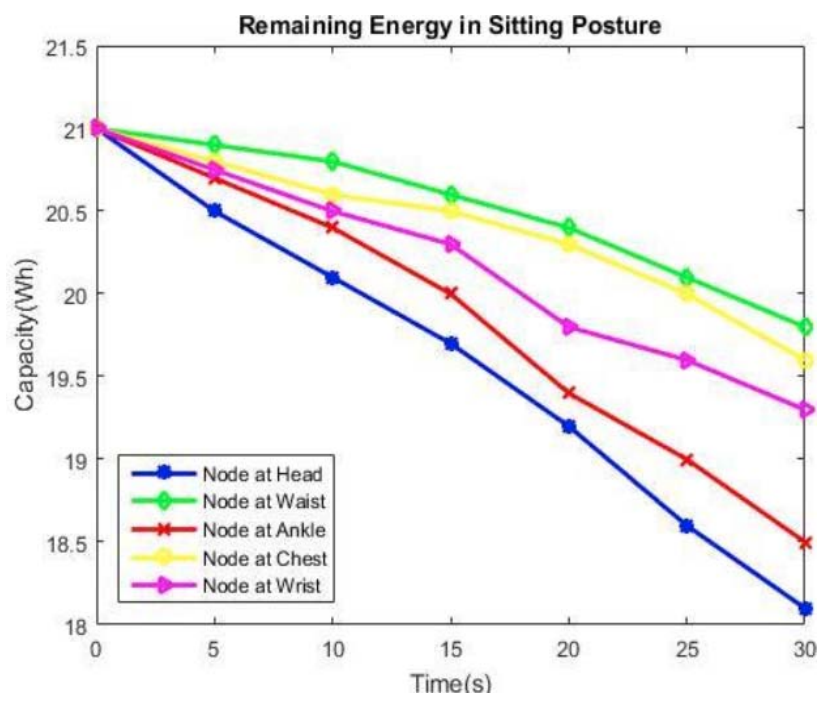

Fig. 11: Energy Consumption in Sitting Posture

\section{Discussion of Results}

From the review in the introduction, it was determined that the selection of suitable sink position can be critical for the performance of a DTN WBAN and that this selection is not a straight forward decision. In this study, a posture selection strategy has been proposed, as outlined in Fig. 6 and the pseudocode. The performance evaluation shows that the average delay has been minimized in the proposed sink locality under the proposed selection strategy under the running and sitting postures. The performance of the different sink node under the various localities, such as the head, ankle, and the waist location in each postural scenario showed that waist location as the best in performance. The delay was recorded to increase as the node moves away from the sink node. The energy consumption showed that the waist position sink node locality has the least energy consumption of the three localities evaluated. This can be related to the fact that nodes far away from waist node have higher mobility hence the reason for high energy consumption.

\section{Vil. Conclusion and Future Work}

The Network lifetime improvement and delay minimization is a key research issue in WBAN. Improper sink selection is one of the main causes of data loss in WBAN due to different posture and WBAN mobility. Various parameters need be observed while selecting the optimal sink position for all the postures. The node with the least mobility and at proximity to the center of body is consider as a sink node. This research proposed a WBAN posture selection strategy which when applied to WBAN with mobility, the best optimal sink node locality is selected. This leads to improved WBAN performance in terms of average WBAN delay and energy consumption. In the evaluation of the sink node selection strategy, it is observed that waist sink 
node locality is the best optimal position as it gives the minimum average delay and energy consumption. In the future, more work should explore mechanisms for dynamic sink node locality to ensure optimal WBAN efficiency.

\section{References Références Referencias}

1. Arefin, M., Ali, M. and Haque, A. (2017) Wireless Body Area Network: An Overview and Various Applications. Journal of Computer and Communications, 5, 53-64. doi: 10.4236/jcc. 2017.57006

2. Bhandari, S., \& Moh, S. (2016). A priority-based adaptive MAC protocol for wireless body area networks. Sensors, 16(3), 401.

3. Chen, W., Hu, J., Bouwstra, S., Oetomo, S. B., \& Feijs, L. (2011). Sensor integration for perinatology research. International Journal of Sensor Networks, 9(1), 38-49.

4. Chen, K. Y., \& DAVID R BASSETT, J. R. (2005). The technology of accelerometry-based activity monitors: current and future. Medicine \& Science in Sports \& Exercise, 37(11), S490-S500.

5. Climent, S., Sanchez, A., Capella, J. V., Meratnia, N., \& Serrano, J. J. (2014). Underwater acoustic wireless sensor networks: advances and future trends in physical, MAC and routing layers. Sensors, 14(1), 795-833.

6. Conan, V., Leguay, J., \& Friedman, T. (2008). Fixed point opportunistic routing in delay tolerant networks. IEEE Journal on Selected Areas in Communications, 26(5).

7. Darkins, A., Ryan, P., Kobb, R., Foster, L., Edmonson, E., Wakefield, B., \& Lancaster, A. E. (2008). Care Coordination/Home Telehealth: the systematic implementation of health informatics, home telehealth, and disease management to support the care of veteran patients with chronic conditions. Telemedicine and e-Health, 14(10), 1118-1126.

8. Gowtham, M., \& Ahila, S. S. (2017). Privacy enhanced data communication protocol for wireless body area network. In Advanced Computing and Communication Systems (ICACCS), 2017 4th International Conference on (pp. 1-5). IEEE.

9. Jaimes, A. F., \& de Sousa, F. R. (2016). A taxonomy for learning, teaching, and assessing wireless body area networks. In Circuits \& Systems (LASCAS), 2016 IEEE 7th Latin American Symposium on (pp. 179-182). IEEE.

10. Jain, S., Fall, K., \& Patra, R. (2004). Routing in a delay tolerant network (Vol. 34, No. 4, pp. 145-158). ACM.

11. Kumar, A., \& Singh, P. (2018). Energy Efficient Transmission Approach for WBAN Based on Threshold distance. Energy, 5(02).
12. Leguay, J., Friedman, T., \& Conan, V. (2007). Evaluating MobySpace-based routing strategies in delay-tolerant networks. Wireless communications and mobile computing, 7(10), 1171-1182.

13. Leguay, J., Friedman, T., \& Conan, V. (2005, August). DTN routing in a mobility pattern space. In Proceedings of the 2005 ACM SIGCOMM workshop on Delay-tolerant networking (pp. 276-283). ACM.

14. Li, L. (2009). Modeling and Analysis on a DTN Based Wireless Sensor Network Topology Control. International Journal of Computer Network and Information Security, 1(1), 32.

15. Li, C., Yuan, X., Yang, L., \& Song, Y. (2015). A hybrid lifetime extended directional approach for WBANs. Sensors, 15(11), 28005-28030.

16. Lindgren, A., Doria, A., \& Schelen, O. (2004). Probabilistic routing in intermittently connected networks. In Service assurance with partial and intermittent resources (pp. 239-254). Springer, Berlin, Heidelberg.

17. Lv, Z., Xia, F., Wu, G., Yao, L., \& Chen, Z. (2010, December). iCare: a mobile health monitoring system for the elderly. In Green Computing and Communications (GreenCom), 2010 IEEE/ACM Int" Conference on \& Int'l Conference on Cyber, Physical and Social Computing (CPSCom) (pp. 699-705). IEEE.

18. Malik, B., \& Singh, V. R. (2013). A survey of research in WBAN for biomedical and scientific applications. Health and Technology, 3(3), 227-235.

19. Mile, A., Okeyo, G., \& Kibe, A. (2018, June). Adaptive Cluster Head Selection Scheme for High Mobility Based IEEE 802.15. 6 Wireless Body Area Networks. Journal of Sensor Technology, 8(02), 35.

20. Mile, A., Okeyo, G., \& Kibe, A. (2018, April). Hybrid IEEE 802.15. 6 Wireless Body Area Networks Interference Mitigation Model for High Mobility Interference Scenarios. Wireless Engineering and Technology, 9(02), 34.

21. Nabi, M., Geilen, M., \& Basten, T. (2011, March). MoBAN: A configurable mobility model for wireless body area networks. In Proceedings of the 4th international ICST conference on simulation tools and techniques (pp. 168-177).

22. Prameela, S., \& Ponmuthuramalingam, P. (2016). A robust energy efficient and secure data dissemination protocol for wireless body area networks. In Advances in Computer Applications (ICACA), IEEE International Conference on (pp. 131134). IEEE.

23. Quwaider, M., \& Biswas, S. (2008). Body posture identification using hidden Markov model with a wearable sensor network. In Proceedings of the ICST 3rd international conference on Body area networks (p. 19). ICST (Institute for Computer Sciences, Social-Informatics and Telecommunications Engineering). 
24. Quwaider, M., \& Biswas, S. (2010). DTN routing in body sensor networks with dynamic postural partitioning. Ad hoc networks, 8(8), 824-841.

25. Quwaider, M., Taghizadeh, M., \& Biswas, S. (2011). Modeling on-body dtn packet routing delay in the presence of postural disconnections. EURASIP journal on wireless communications and networking, 2011, 3.

26. Sipal, V., Gaetano, D., McEvoy, P., \& Ammann, M. J. (2015). Impact of hub location on the performance of wireless body area networks for fitness applications. IEEE Antennas and Wireless Propagation Letters, 14, 1522-1525.

27. Spyropoulos, T., Psounis, K., \& Raghavendra, C. S. (2008). Efficient routing in intermittently connected mobile networks: The multiple-copy case. IEEE/ACM transactions on networking, 16(1), 77-90.

28. Spyropoulos, T., Psounis, K., \& Raghavendra, C. S. (2008b). Efficient routing in intermittently connected mobile networks: The single-copy case. IEEE/ACM transactions on networking, 16(1), 63-76.

29. ul Huque, M. T. I., Munasinghe, K. S., \& Jamalipour, A. (2015). Body node coordinator placement algorithms for wireless body area networks. IEEE internet of things journal, 2(1), 94-102.

30. Varga, A. (2001, June). Discrete event simulation system. In Proc. of the European Simulation Multiconference (ESM'2001).

31. Yi, Z., Shan, Z., Wang, J., Li, J., Cheng, C., Haoting, X., ... \& Xiao, S. (2016). Ubiquitous healthcare system using emergency strategy based on wireless body area system. In Computer Science and Network Technology (ICCSNT), 2016 5th International Conference on (pp. 117-120). IEEE. 